\title{
Health economic evaluations of non-pharmacological interventions for persons with dementia and their informal caregivers: a systematic review
}

Franziska Nickel ${ }^{1,2^{*}}$ (D) Janina Barth ${ }^{1,2}$ and Peter L. Kolominsky-Rabas ${ }^{1,2}$

\begin{abstract}
Background: This systematic review aims to review the literature on trial-based economic evaluations of non-pharmacological interventions directly targeted at persons with dementia as well as persons with mild cognitive impairment and their respective caregivers.

Methods: A systematic literature research was conducted for the timeframe from 2010 to 2016 in the following databases: Centre for Reviews and Dissemination, EconLit, Embase, Cochrane Library, PsycINFO and PubMed. Study quality was assessed according to the Drummond criteria.

Results: In total sixteen publications were identified. Health economic evaluations indicated the cost-effectiveness of physical exercise interventions and occupational therapy. There was also evidence to suggest that psychological and behavioral therapies are cost-effective. Health economic studies investigating psychosocial interventions mainly targeted towards informal caregivers showed inconsistent results.
\end{abstract}

Conclusions: Due to the increasing prevalence of dementia non-pharmacological interventions and their health economic impact are of increasing importance for health care decision-makers and HTA agencies.

Keywords: Dementia, Non-pharmacological interventions, Economic evaluation, Costs, Cost-effectiveness

\section{Background}

Estimates show that there are around 47 million people living with dementia (PwD) worldwide [1]. In 2015, the global societal economic cost was estimated to be US\$818 billion [1]. As the prevalence of dementia is projected to increase substantially, it poses significant challenges to health and social care systems [1].

Regarding treatment options, acetylcholinesterase inhibitors provide small but clinically important symptomatic benefits on cognition and function for persons with Alzheimer's disease (AD), the most prevalent subtype of dementia, persons with Lewy body dementia and Parkinson's disease dementia, without counteracting the

\footnotetext{
* Correspondence: Franziska.Nickel@uk-erlangen.de

${ }^{1}$ Interdisciplinary Centre for Health Technology Assessment (HTA) and Public Health (IZPH), Friedrich-Alexander-University Erlangen-Nürnberg (FAU),

Schwabachanlage 6, 91054 Erlangen, Germany

${ }^{2}$ National Graduate College 'Optimisation strategies in Dementia - OptiDem', Karl and Veronica Carstens-Foundation, Essen, Germany
}

progression of the disease [2-4]. Moreover, in persons with moderate-to-severe $\mathrm{AD}$ memantine has a small effect on cognition. However, these pharmacological interventions may provoke side-effects [4].

Furthermore, over the last decade non-pharmacological interventions for PwDs and persons with mild cognitive impairment (PwMCI) as well as their caregivers became more important, leading to a considerable growth in the evidence base $[5,6]$.

Results of meta-analyses indicate that physical exercise interventions may have a positive effect on the rate of cognitive decline in $\mathrm{AD}[7]$ and suggest that there is a beneficial effect on cognitive functioning independent of the subtype of dementia [8]. In contrast, a review of the Cochrane Collaboration [9] did not find clear evidence on the benefit of physical exercise programs on cognition of PwDs. The Cochrane meta-analysis showed that there may be a significant benefit from physical exercise interventions on 
the ability to perform activities of daily living in PwDs, but the rated quality of evidence was very low [9].

Further evidence suggests that psychological interventions can reduce symptoms of depression and anxiety among PwDs [10]. This is of particular importance, since depressive symptoms are common in all types and disease stages of dementia as well as in PwMCI and may impact considerably on the quality of life of PwDs and their caregivers [11]. With regard to caregiver interventions, a metaanalysis of high-quality RCTs shows that multicomponent interventions based on education and support elements delay the institutionalization of persons with $\mathrm{AD}$, degenerative or mixed dementias [12].

Previously performed systematic reviews highlight the scarcity of economic evidence regarding non-pharmacological interventions for PwDs [13] and their supporting informal caregivers [14]. However, to inform resource allocation decisions, information on effective and cost-effective intervention strategies is essential for governmental decision-makers or payers [15].

Hence, the aim of this article is to conduct a systematic review of recent trial-based economic evaluations and cost studies of non-pharmacological interventions directly targeted at PwDs, PwMCI or their informal caregivers.

\section{Methods}

\section{Search strategy}

A systematic literature review was performed, searching the following key databases: Centre for Reviews and Dissemination (National Health Service Economic Evaluation Database; Health Technology Assessment), Cochrane Library, EconLit (EBSCO), Embase (OvidSP), PsycINFO (EBSCO) and PubMed (Medline). The timeframe for electronic searches was restricted to publications from 2010 to 2016. Key words concerning the dementia syndrome such as "dementia", "Alzheimer's Disease" etc. were combined with search terms in the context of economic evidence as, for instance, "cost", "economic evidence", "cost-utility" "cost-effectiveness" and "savings". The complete research strategies for the respective databases are presented in the Additional file 1.
Unpublished or grey literature was not included. The search was limited to studies in English or German.

\section{Study selection}

Study eligibility was based on title and abstract screening, performed by two independent reviewers. A third independent reviewer was consulted in any case of disagreement. Articles passing the initial screening were retrieved for a detailed full text evaluation. The selection criteria are presented in Table 1.

This review includes studies based on standard economic evaluation methods. Cost-effectiveness analysis (CEA) measures and compares consequences in terms of an appropriate single natural effect or effect on a physical unit, which is common to all considered alternatives. The ratio of the mean incremental cost and the mean incremental effect of the interventions under evaluation is called incremental cost-effectiveness ratio (ICER). The ICER represents a summary measure that may support the decision making process by indicating the cost per unit change with respect to the evaluated outcome [15].

By contrast, cost-utility analysis (CUA) enables a comparison of multiple effects that are not necessarily common to the evaluated alternatives, as multi-dimensional health outcomes are captured into a single index [16]. In CUA health state preference scores (utility values) are used to value the states of health associated with the respective consequences of the interventions. Quality-adjusted life-years (QALY), which combine morbidity and mortality are the most frequently employed outcome measure in CUA [15].

\section{Data extraction}

Data extraction was performed in line with the recommendation of the Centre for Reviews and Dissemination for reviews of economic evaluations [17], including type of economic evaluation, study objective, description of the intervention and comparators, measure of benefit, cost data and respective sources, methods for dealing with uncertainty as well as cost and outcome results.

Table 1 Inclusion and exclusion criteria

\begin{tabular}{lc}
\hline Inclusion criteria & Exclusion criteria \\
\hline - Solely trial-based, non-modelling economic studies & - Model-based economic studies were excluded. \\
were included. & - Studies evaluating organizational changes and changes \\
- The study design had to compare cost outcomes of & in thelivery of care and support were not considered. \\
non-pharmacological interventions directly targeted & Qualitative research, cost-of-illness studies, case studies, \\
at persons with dementia, persons with mild cognitive & studies without a control group, systematic reviews or \\
impairment or the respective informal caregiver. & meta-analyses were excluded. \\
- The presence of an independent control group. & abstracts were not considered. \\
- No restriction was made regarding the type or stage & \\
- - The dementia. & \\
and published between 01.01 .2010 and 31.12 .2016$. &
\end{tabular}




\section{Quality appraisal}

The appraisal of study quality was based on Drummond's ten-item check-list for assessing economic evaluations [see Additional file 2] [15].

\section{Results}

\section{Study selection}

The systematic literature search identified 10,047 publications [see Additional file 1]. After the removal of duplicates, title screening of 6,835 articles was conducted. Subsequent to the screening of 390 abstracts, 37 articles were retrieved in order to assess the full texts. Of these, 16 were included in the synthesis. The flow of articles retrieved through electronic searches is depicted in Fig. 1.

\section{Basic characteristics of the included studies}

The largest part of the included studies was conducted in the United Kingdom (seven studies). Of these, two articles are based on results from the British START (STrAtegies for RelaTives) study [18, 19]. Three studies were from the United States of America and two were from Finland. Further, one study each was conducted in Canada, the Netherlands, Denmark and Sweden.
Two articles report data on interventions that are mainly targeted at PwDs [20, 21], whereas seven studies comprise interventions, which involve both the $\mathrm{PwD}$ and the respective caregiver [22-28]. Six publications deal with programs that assist caregivers of PwDs [18, 19, 29-32]. Solely one study was targeted at PwMCI [33].

\section{Synthesis}

The identified studies were grouped based on a classification proposed in the World Alzheimer Report 2011 [34].

Non-pharmacological interventions addressed to PwDs and, if applicable, to the patient-caregiver dyads were classified into the following categories:

i.) Physical exercise interventions;

ii.) Interventions to support and enhance cognitive abilities in PwDs as for instance reality orientation, reminiscence therapy or cognitive stimulation;

iii.) Psychological and behavioral therapies;

iv.) Occupational therapy.

Moreover, interventions that primarily target caregivers were classed together.

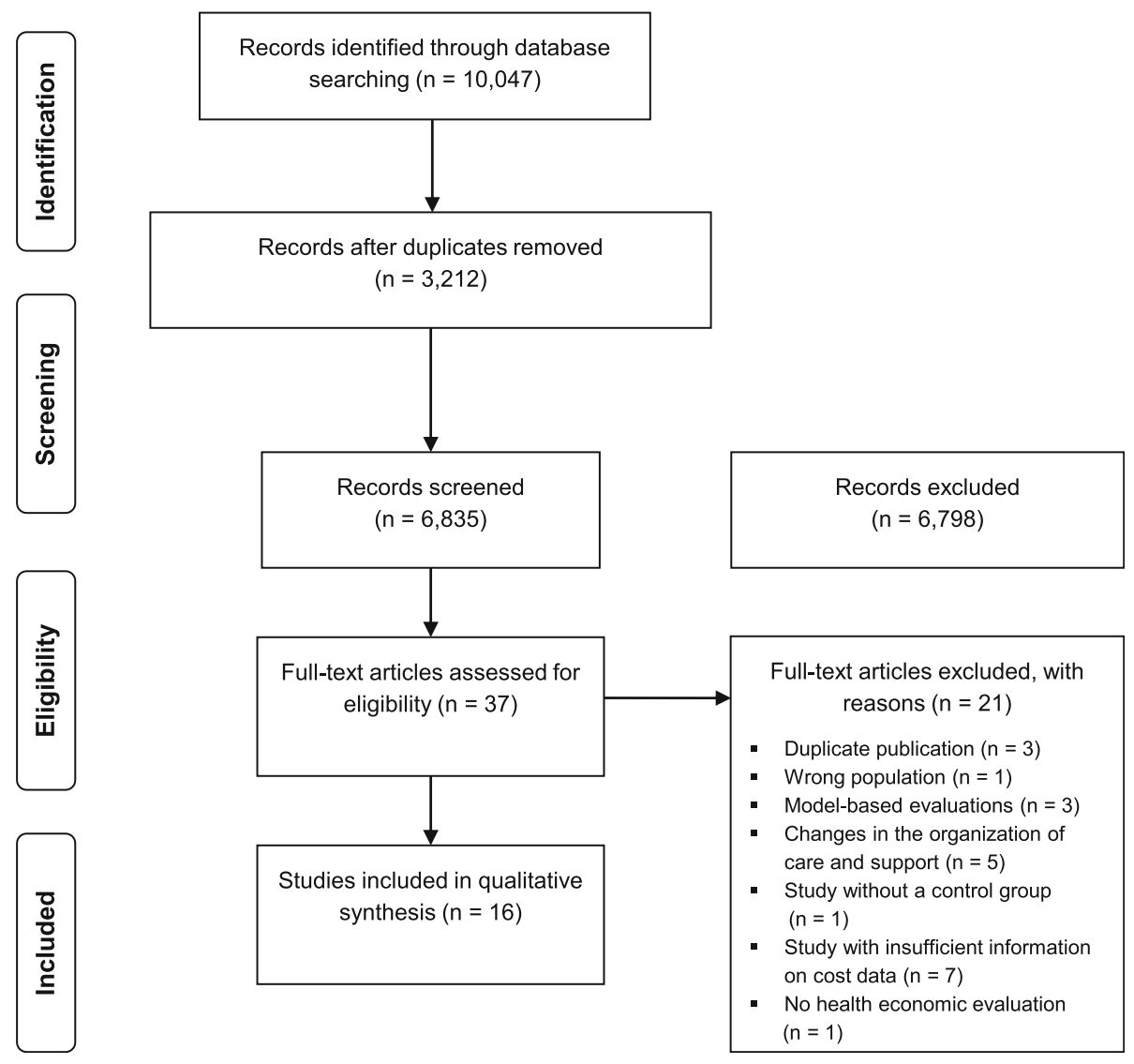

Fig. 1 PRISMA flow chart 


\section{Physical exercise interventions}

Two trial-based CEA evaluating physical exercise interventions for PwDs [20,28] and one for PwMCI were identified [33]. The exercise programs of the reviewed CEAs pursue different therapeutic goals, namely the reduction of behavioral and psychological symptoms of dementia (BPSD) [28] or the delay in the deterioration of patient's physical functioning [20]. The intervention evaluated by Davis et al. [33] aims to improve executive cognitive functions in PwMCI. These different therapeutic goals are also reflected in the range of employed effectiveness measures (see Table 2).

In Finland a CEA was conducted concurrently with the Finnish Alzheimer disease exercise trial (FINALEX) [20]. In the first study arm a one-year group exercise program for community-dwelling persons with $\mathrm{AD}$ provided twice a week during visits to day care centers was assessed. The second arm included a one-year tailored home-based exercise program administered twice a week by physiotherapists. Both interventions were compared to a control group obtaining care as usual (CAU). To measure the impact of these intense and long term exercise programs on the physical functioning and mobility of the persons with $\mathrm{AD}$, the Functional Independence Measure (FIM) and the Short Physical Performance Battery (SPPB) were used. Health outcomes were measured at baseline and after three, six and 12 months. Cost data was retrieved for the patientcaregiver dyads from central registries and medical records over a period of 24 months. Pitkälä and colleagues [20] came to the conclusion that an intensive and long-term exercise program administered in the patient's home could slow the decline in physical functioning without increasing total health and social service costs.

A CEA conducted by D'Amico et al. [28] evaluated an intervention targeting community-dwelling PwDs who showed at least one BPSD. The intervention under evaluation was composed of a 12-week daily walking program (lasting for 20 to $30 \mathrm{~min}$ ) performed by the patient-caregiver dyad in the surrounding of their residence. The dyads were instructed by a qualified exercise professional. The program was designed to become increasingly more intense, whereas the control group received CAU. The reduction in BPSDs was measured by means of the Neuropsychiatric Inventory (NPI) as the primary effectiveness outcome. Secondary analyses considered the impact on quality of life of the participants with dementia (measured using the DEMQOLProxy), caregiver burden (collected by employing the Zarit Caregiver Burden Interview (ZBI)) and caregivers' mental health (measured using the General Health Questionnaire (GHQ)). Further, QALYs were estimated based on DEMQOL-Proxy scores. A subsample of 52 dyads was analyzed within the framework of the economic analyses [28]. In contrast to the entire sample [35], the economic subsample indicated a statistically significant between-group difference considering the average scores of the GHQ at 12 weeks, favoring the intervention group. The other evaluated outcomes did not show a significant difference at follow-up. The Client Service Receipt Inventory (CSRI) was completed by the caregiver in order to gather data on resource utilization. From the viewpoint of the health and social care system, the intervention group showed lower costs compared to the CAU control group, hence the intervention was dominant; however these results were not statistically significant. From a societal viewpoint, the ICER was estimated to amount to $£ 421$ per unit change in the NPI score. Assuming that a reduction of at least three points in the NPI score would be considered clinically relevant, the authors derived the suggestion that a clinically meaningful improvement could be achieved at a cost of $£ 1,263$. Therefore D'Amico et al. [28] concluded that the individually tailored walking program is potentially cost-effective when considering BPSD; however they also point to the fact that for the NPI no cost-effectiveness threshold was yet defined. The ICER for QALY was high, thus the intervention seems not to be cost-effective with regard to QALY gains.

In a Canadian three-arm trial, Davis et al. [33] assessed the cost-effectiveness of resistance training and aerobic training compared to a control group receiving balance and tone classes targeted at community dwelling women aged 70 to 80 , who self-reportedly expressed to experience memory problems. As primary effectiveness outcome measure the executive cognitive function of selective attention and conflict resolution was assessed by means of the Stroop Test. From the point of view of the Canadian healthcare system, the incremental cost per incremental Stroop change score showed that aerobic training as well as resistance training decreased healthcare costs and proved to be more effective than twice weekly balance and tone classes. The study was limited by the fact that the participants did not have a formal mild cognitive impairment diagnosis. Moreover, the resource use data was collected through a self-completed questionnaire, which might have caused a recall bias.

\section{Interventions to support and enhance cognitive function}

Three British RCTs were included that evaluated strategies to support and enhance cognition in PwDs [21, 26, 27]. The main considered outcomes were cognition and health-related quality of life (see Table 3).

Orgeta et al. [27] assessed structured, individual, homebased cognitive stimulation sessions for PwDs, administered by the respective caregiver. The caregivers were trained at home by researchers who also provided support via additional home visits or via telephone. Carers received a range of materials such as a manual, an activity workbook and a toolkit comprising boules and playing cards. The program was composed of 75 themed activity sessions. The sessions were designed to last $30 \mathrm{~min}$ and were scheduled to be delivered three times a week, over a period of 


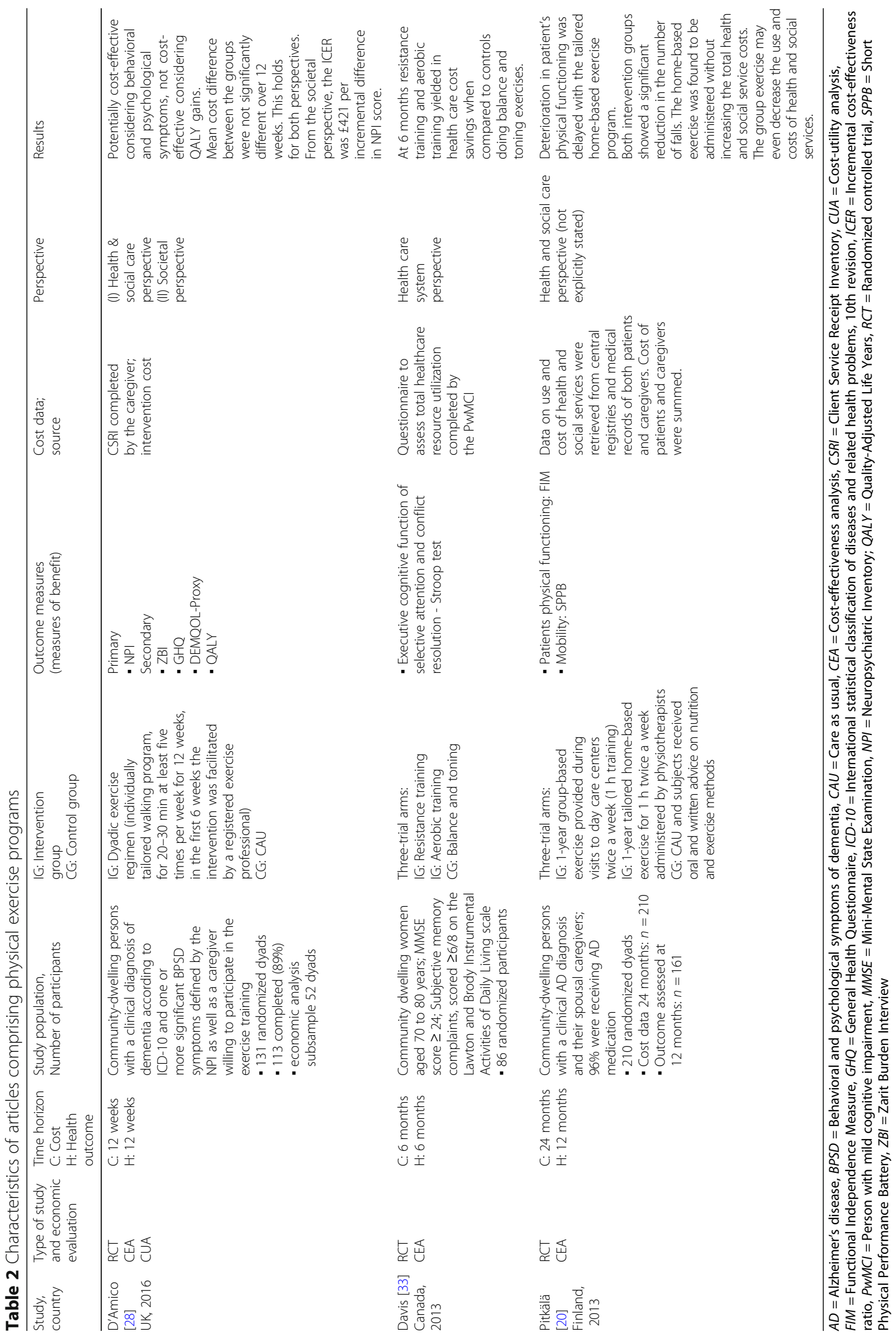




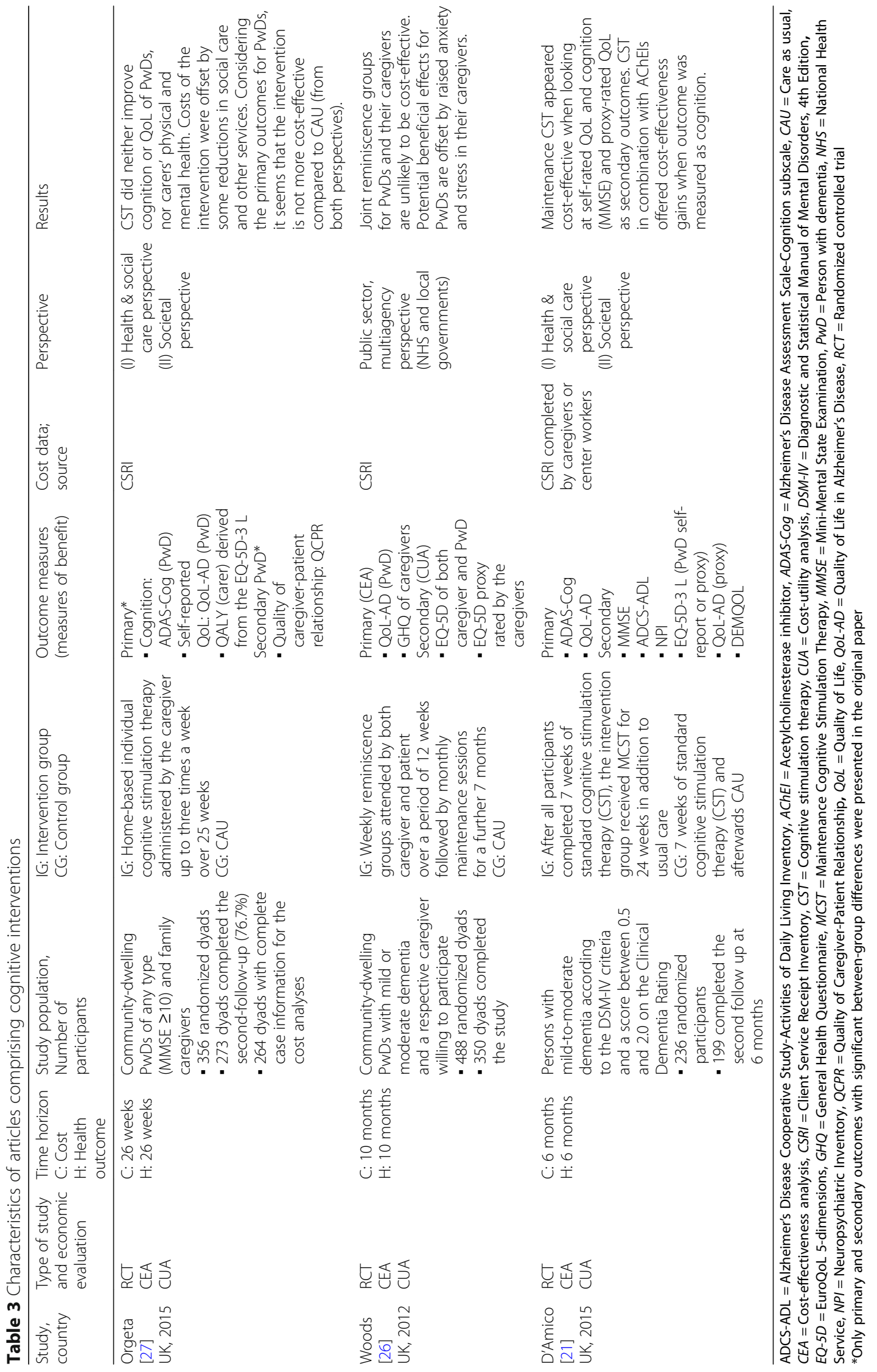


25 weeks. Compared to treatment as usual, the individual cognitive stimulation therapy (iCST) did not significantly improve cognition (measured by the Alzheimer's Disease Assessment Scale-Cognition subscale (ADAS-Cog)) or quality of life of the PwD (assessed by the Quality of Life in Alzheimer's Disease scale (QoL-AD)). This also applies for the caregivers' physical and mental health (assessed by the Short Form questionnaire-12 items (SF-12)). Intervention costs were offset by some reductions in social care and other services. Even though improvements were neither observed with regard to most of the secondary outcomes for PwDs (activities of daily living, depression and BPSD), analyses indicated that PwDs in the intervention group may experience a higher relationship quality with their caregiver. From the health and social care perspective as well as from the societal perspective, there was no significant betweengroup difference with regard to mean costs. The authors concluded that considering the primary outcomes, from either perspective, the structured home-based cognitive stimulation does not seem to be more cost-effective than CAU. Further, the researchers remark that there are no available societal willingness-to-pay thresholds for the improvement in the primary outcomes measured by ADASCog, QoL-AD or the Quality of Caregiver-Patient Relationship (QCPR) instrument. Considering the QALY gain for caregivers derived from the EQ-5D with societal weights, iCST was found to be more effective than CAU.

In another trial D'Amico et al. [21] evaluated the costeffectiveness of a weekly maintenance cognitive stimulation therapy (MCST) over a period of 24 weeks compared to treatment as usual. Before entering the study, all participants completed seven weeks of twice-weekly standard cognitive stimulation therapy [36]. At six months, no difference between the control and intervention group was found with regard to the primary outcome cognition (measured by ADAS-Cog). However, the second primary outcome self-rated quality of life (assessed by means of the QoL-AD) was found to be higher in the intervention group compared to the CAU group. Participants receiving CAU showed only slightly non-significantly lower health and social care costs than participants in the MCST intervention group. The estimated ICER demonstrates that the mean cost for a 1 point difference on the QoL-AD scale was $£ 266$. The respective cost-effectiveness acceptability curve (CEAC) indicates that MCST can be considered cost-effective with a probability of $90 \%$ at a willingness-topay of around $£ 1,400$. In contrast, this probability was low considering cognition as outcome. Even though no significant between-group difference on the Mini-Mental Status Examination (MMSE) score was observed, the CEA indicated that MCST is likely to be cost-effective considering MMSE as outcome measure. Considering QALY gains derived from EQ-5D proxy-ratings, the mean incremental cost-utility ratio (ICUR) was $£ 26,835$. At a threshold of
$£ 20,000$, CEAC suggests that considering the former outcome the probability of cost-effectiveness was $40 \%$. Sensitivity analyses conducted from the societal perspective yielded inconsistent cost-effectiveness findings.

Within the framework of the REMCARE trial Woods et al. [26] evaluated the cost-effectiveness of weekly joint reminiscence groups provided for PwDs and their caregivers over a time span of 3 months, followed by monthly maintenance sessions over a period of 7 months. Primary outcomes were the PwD's quality of life (measured by the QoL-AD) and carer's mental health (assessed by the General Health Questionnaire-28 (GHQ-28)). With regard to these primary outcomes neither after 3 months nor after 10 months significant group-difference could be found. After 10 months, caregivers assigned to the intervention group even showed a significant increase in anxiety. Considering the mean health and social service costs for PwDs, the mean total costs for the intervention group was found to be $13.5 \%$ higher compared to the control group. However this betweengroup difference was not statistically significant. The estimated ICER for the QoL-AD was $£ 2,586$ (CI: $-20,280$ to 24,340). Woods et al. [26] concluded that the intervention was neither effective nor cost-effective.

\section{Psychological and behavioral interventions}

Three publications evaluated psychological and behavioral therapies delivered to PwD-caregiver dyads (see Table 4) $[22,24,25]$.

A pilot RCT conducted by Spector et al. [25] in the UK assessed the cost of a ten-session cognitive-behavioral therapy (CBT) for patient-caregiver dyads compared to treatment as usual from the health and social care perspective. The Rating Anxiety in Dementia scale (RAID) was used as the primary outcome measure. The cost analysis yielded that at 15 weeks the adjusted difference in anxiety of the PwDs was lower in the CBT group compared to the control group (-3.10; 95\% CI: -6.55 to 0.34 ). This finding was maintained at 6 months ( -4.59 ; 95\% CI: -9.34 to 0.15$)$. Further, at 15 weeks depression of the PwDs measured by the adjusted Cornell Scale for Depression in Dementia (CSDD) was lower in the CBT group (-5.37; 95\% CI: -9.50 to -1.25$)$, which remained significant at 6 months. The total cost that occurred over the 6-month follow-up period were found to be significantly lower within the CBT group compared to CAU (adjusted mean difference - $£ 564.38 \quad(95 \% \quad \mathrm{CI}$ : $-£ 1,252.08$ to $-£ 112.85)$ ). While also including intervention cost, estimated to amount to $£ 1,002$ per person, the total cost from the health and social service perspective were lower for the control group, with an adjusted mean difference of $£ 769.80$ (95\% CI: $-£ 121.99$ to $£ 1,697.38$ ). However, this difference was not found to be statistically significant. Hence, the authors concluded that CBT proved to be cost neutral. 


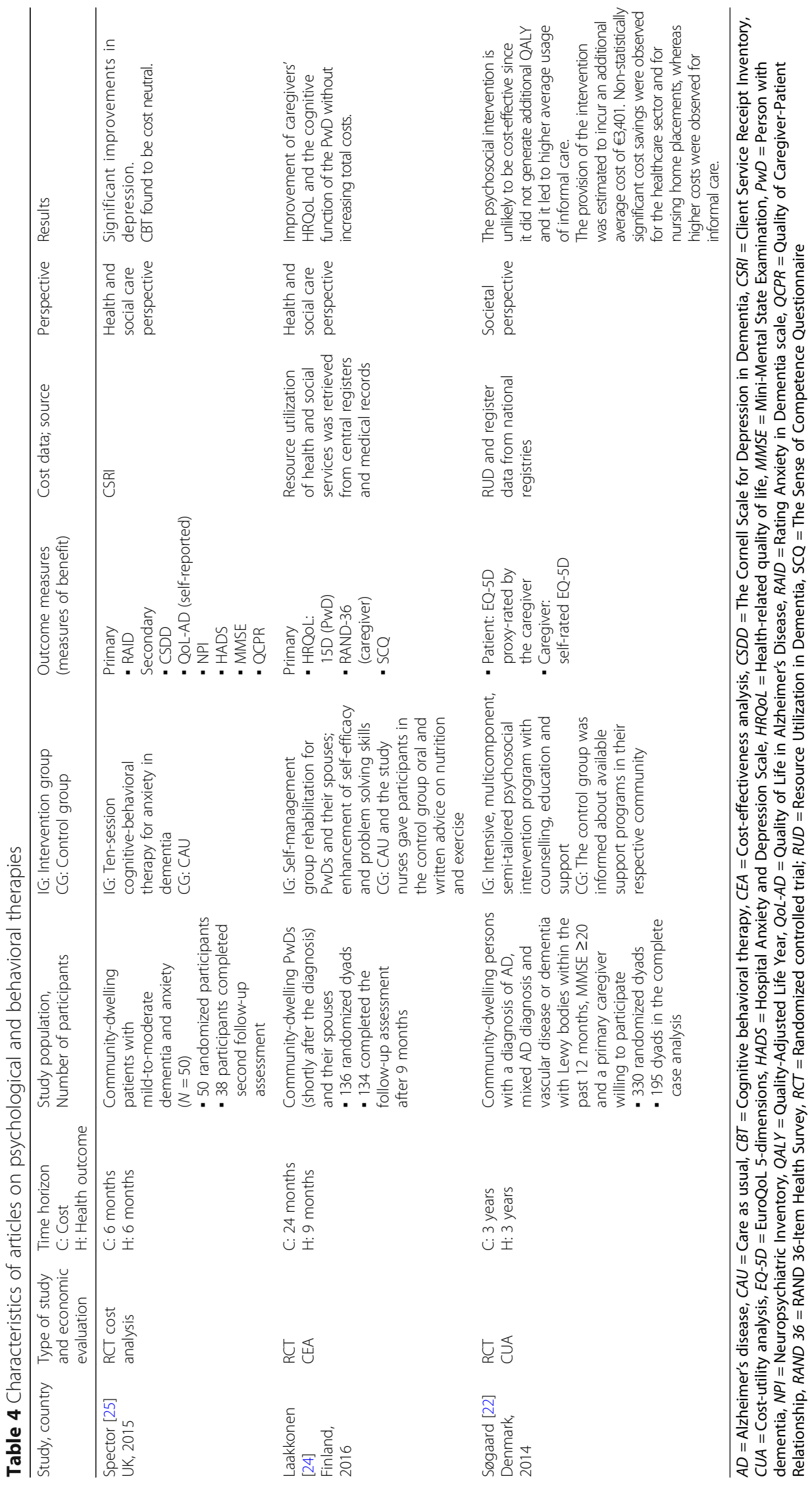


A Finnish trial conducted by Laakkonen et al. [24] performed a CEA, evaluating a self-management group rehabilitation program for PwDs and their spouses administered shortly after the diagnosis. The intervention comprised eight group sessions of self-management offered to the spousal dyads and was compared to CAU. At 3month, the intervention group demonstrated higher HRQoL outcomes as measured with the Finnish version of the RAND 36-Item Health Survey in spouses of PwD, however after 9 months the effect was weakened. With respect to spouses' sense of competence and feelings of mastery (assessed by the Sense of Competence Questionnaire (SCQ)) no significant group difference was demonstrated. Further, cognitive scores of PwDs in the intervention group improved significantly more than those of the control group. These positive effects were not accompanied with higher health and social services costs; hence the authors concluded that the self-management group rehabilitation is cost neutral.

As part of the Danish Alzheimer's Intervention Study, which assessed the provision of an early psychosocial intervention for community-dwelling PwDs with a recent diagnosis and their respective caregivers, a CUA was conducted from the societal perspective. Søgaard and his colleagues [22] evaluated an intensive, multicomponent, semi-tailored psychosocial intervention program over a period of 3 years. The program was composed of counselling, educational and support elements, which were compared to a control group that was informed about available support programs in their respective communities [37]. QALY were estimated individually for the $\mathrm{PwD}$ and the respective caregiver and eventually aggregated for the analysis. HRQoL values of the PwDs were measured by means of proxy-rated EQ-5D. Cost data on informal care and production loss was collected by means of the Resource Utilization in Dementia (RUD) instrument. Data on resource utilization in the primary and secondary health sector was based on national registers. Within both study arms, no significant difference was observed between QALYs and cost measures. The observed cost increase with respect to informal care was not outweighed by the savings in the formal care sector [22].

\section{Occupational therapy}

Gitlin et al. [23] examined the cost-effectiveness of a structured intervention administered by an occupational therapist composed of six in-home sessions and two telephone contacts over a period of 4 months (see Table 5). The intervention was structured into 3 parts: firstly the capabilities of the PwD were assessed. Secondly, an identification of activities that are tailored to the prevailing capabilities of the $\mathrm{PwD}$ was conducted and caregivers were instructed on support strategies. Eventually, when the activities were mastered, the occupational therapist was giving advice on how to employ the practiced techniques to other care challenges. The intervention reduced the time the caregiver was occupied by activities provided in relation to the PwD and was found to be cost-effective compared to treatment as usual.

\section{Psychosocial interventions mainly targeted at the caregiver}

Six publications were found that focused on five interventions mainly delivered to informal caregivers of PwDs (see Table 6) [18, 19, 29-32].

A Dutch CEA and CUA conducted by Joling et al. [29] evaluated the effectiveness of a family meetings intervention on caregiver's mental health compared to CAU from the societal perspective. Data was gathered alongside a RCT of 192 primary caregivers of community-dwelling PwD. The intervention consisted of two individual meetings between the caregiver and a trained counsellor and four structured family meetings with additional relatives and friends joining in. Apart from the scheduled sessions, the counsellor was available for support via telephone. The objective of the family meetings was to provide psychoeducation, problem solving techniques and to mobilize the existing family networks. However, after 12 months, significant effects on caregiver's depression and anxiety could not be observed. The same applies for caregiver's and patient's quality of life. Further, over the 12 months, no significant cost difference between the intervention and control group was measured. Total cost per patientcaregiver dyad was estimated to be EUR 77,832 in the family meetings group compared to EUR 75,201 in the CAU group (mean adjusted difference EUR 4,149; 95\% CI: $-13,371$ to 21,956 ; ICER 157,534 ). Hence, the researchers drew the conclusion that compared to CAU the provision of family meetings cannot be considered a cost-effective strategy.

A Swedish CUA by Dahlrup et al. [30] evaluated the cost-effectiveness of a psychosocial intervention for family caregivers of PwDs including five weekly counselling sessions administered by a registered nurse and a counselor. Education and information on dementia as well as available services in the community were followed by a group discussion. Caregivers who were unable to join group sessions received individual meetings and were invited to continue the support groups twice a month over a period of three months. 12 months after the fifth educational session, a follow-up was offered for each group. Over the time span of the study, caregivers in the intervention group were given the opportunity to contact a physician, the administering nurse or the counselor. With regard to the endpoints survival and time to institutionalization of the $\mathrm{PwD}$, the intervention group did not differ significantly from the control group with CAU. A subgroup analysis based on the relation between caregiver and PwD was conducted, which demonstrated that if the caregiver was a spouse or cohabitant, PwDs transition into the 


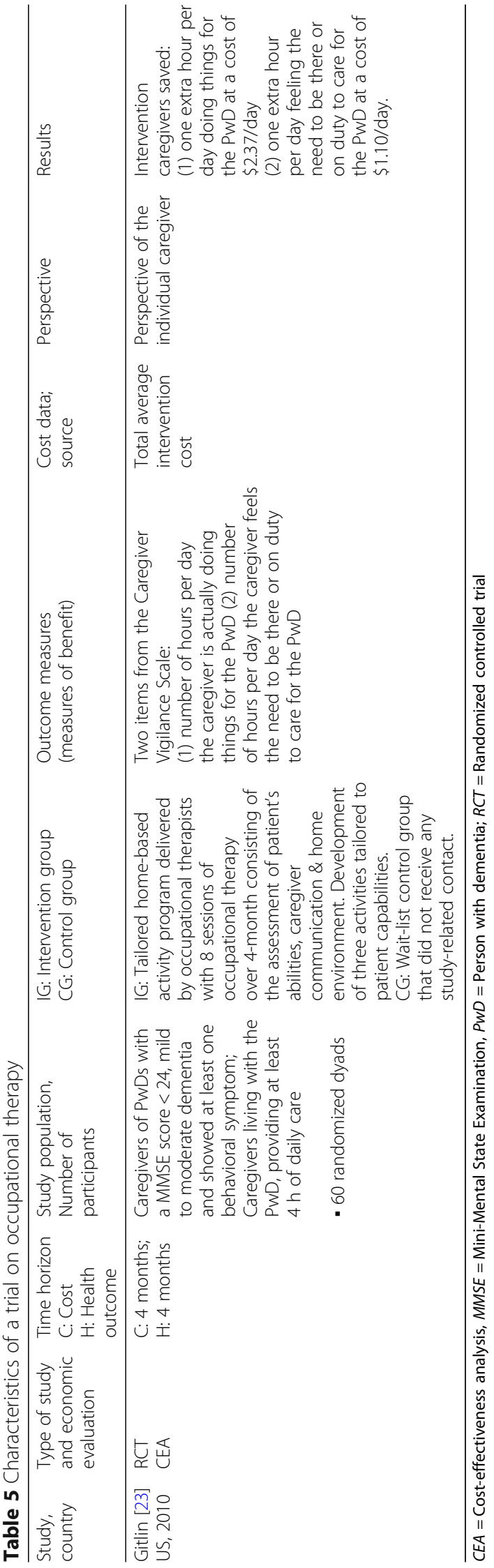




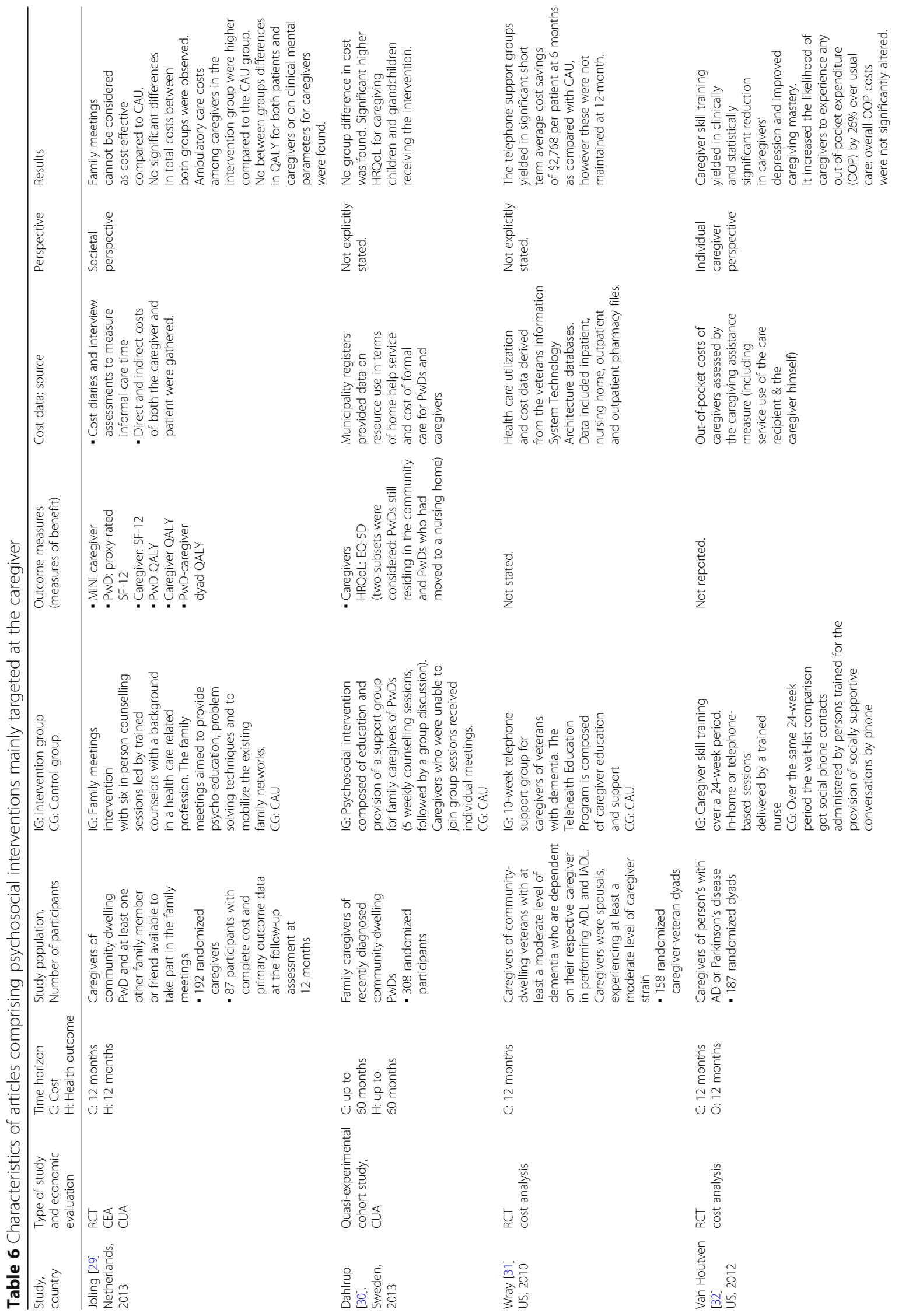




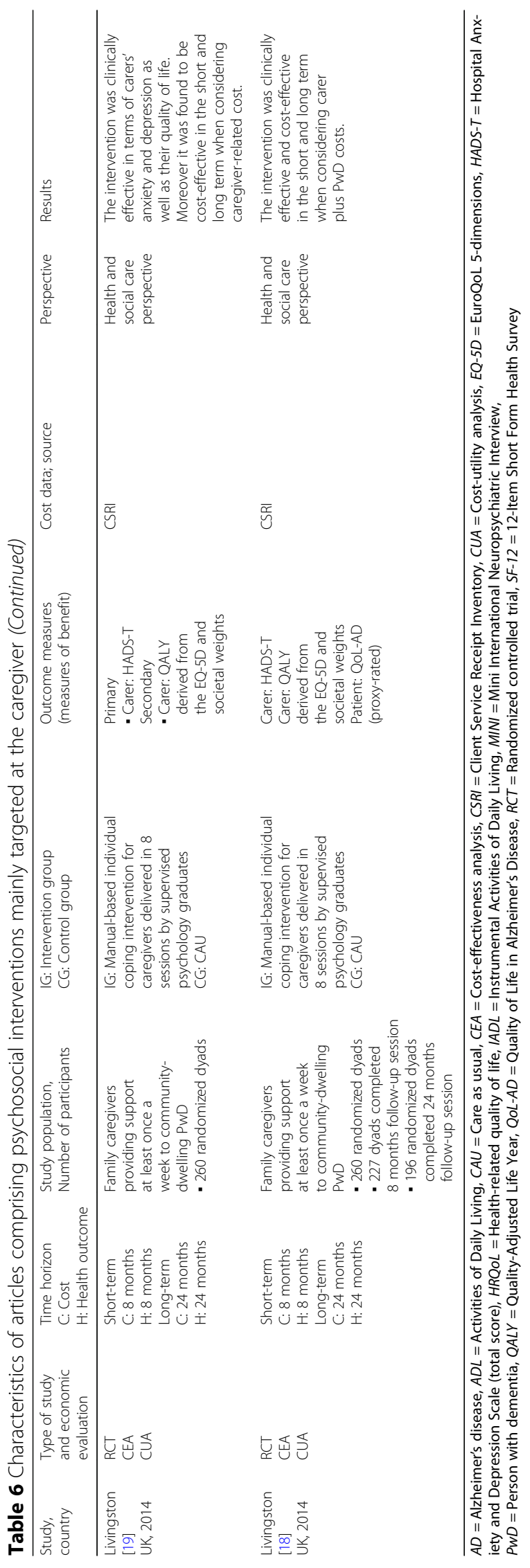


nursing home occurred earlier compared to the control group $(p<0.01)$. In case the caregiver was a child or grandchild of a PwD, patients in the intervention group stayed longer in the community compared to controls $(p=0.06)$. The analysis of caregiver's Health Related Quality of Life (HRQoL) was distinguished into caregivers whose care recipient was still living in the community and PwDs that had moved to a nursing home. For the former subgroup, caregiver's health-related quality of life measured by EQ-5D was significantly higher in the intervention group compared to the controls. There was no significant in between-group difference with regard to caregivers whose care recipient moved to a nursing home. Depending on the caregiver's relation to the care recipient, HRQoL differed. Resource use for home help services, nursing home care and the study intervention were collected. The cost analysis accounted for the differing length of observation. Over the 5-year follow-up, the median total cost weighted for study length did not differ between the two groups under consideration. For the intervention group the median total cost per month amounted to EUR 1,926 (interquartile range 1,043 to 2,588) in comparison to EUR 1,860 (interquartile range 864 to $2,577)$ in the control group $(p=0.47)$. Hence, it can be concluded that the intervention yielded significantly higher HRQoL in children and grandchildren caring for a PwD without significantly increasing the evaluated cost (i.e. costs for home help services, nursing home placement and the intervention cost).

An American cost analysis performed by van Houtven et al. [32] evaluated the effect of skill training aiming to reduce depressive symptoms of family caregivers of persons with $\mathrm{AD}$ or Parkinson's disease on the caregiver's out-of-pocket (OOP) costs. The skill training lasted over 24 weeks and included in-home or telephone-based sessions administered by a trained nurse. The control group was composed of the wait-list participants, who received supportive consultations via phone. Van Houtven and colleagues [32] reported clinically and statistically significant reductions in depressive symptoms and positive effects on caregiving mastery for the participants in the intervention group. The cost analysis solely included OOP costs faced by the caregiver for services provided to the care recipient or the caregiver himself. The analysis indicates that the intervention increased the likelihood of caregiver's OOP expenditure by $26 \%$ points, however no intervention effect was observed with regard to the overall level of expenditures. It was acknowledged by the authors that OOP cost form only a part of the total economic cost which caregivers may face [32].

Wray et al. [31] examined the effect of the provision of a telehealth education program to spousals of veterans with dementia in the United States of America. The telephone support group included educational aspects on the syndrome as well as training on emotion-focused and problem-focused coping strategies and was administered over a period of ten weeks. Health care utilization and cost data were retrieved from the veterans' databases. The cost analyses showed that in the short-term, 6 months after the intervention start, the total health care costs were significantly lower in the intervention arm in comparison to the CAU control $(p=0.039)$. The same applies when separately considering nursing home cost $(p=0.009)$. Mean overall cost savings achieved in the intervention group amounted to $\$ 2,768$ per PwD. Of these, mean nursing home cost savings per PwD were $\$ 1,059$. However, over the 12-month follow-up, the decrease in total care cost was not maintained.

Livingston and colleagues [19] evaluated the short and long-term cost-effectiveness of a manual-based individual coping strategy intervention (START) targeted at family caregivers of PwD in the UK. The manual was based on the Coping with Caregiving intervention developed in the US. The START intervention consisted of eight psychological therapy sessions, administered by psychological graduates. The sessions covered psychoeducation about dementia, caregiver stress and behavior strategies [38]. The short-term analysis over a period of 8 months with QALYs as outcome measure yielded that caregivers receiving the intervention showed higher, however non-significant, health and social care costs amounting to $£ 252$ (95\% CI: $£ 28$ to £565) compared to CAU and an incremental QALY gain of 0.042 (95\% CI: 0.015 to 0.071 ). With the total score of the Hospital Anxiety and Depression Scale (HADS-T) as outcome the incremental health and social care costs were estimated to be $£ 247$ ( $95 \%$ CI: $£ 0$ to $£ 569$ ) higher in the START group. The ICUR was $£ 6,000$ per additional QALY and $£ 118$ per unit change on HADS-T. The likelihood that the START intervention was cost-effective at a willingness-to-pay threshold of $£ 20,000$ was $93 \%$. In the long-term (after 24 month) for the QALY analysis, the cost per additional QALY was $£ 11,200$ and the CEAC indicated that the likelihood that the intervention was cost-effective was $75 \%$ presuming a willingness-to-pay threshold of $£ 30,000$. The authors concluded that the START intervention was costeffective over 8 and 24 months.

A second publication of Livingston et al. [18] additionally assessed PwD's outcomes and costs. The analyses showed that when combining carer and PwD's costs, the START intervention also dominates CAU considering caregiver's outcomes. However, with regard to PwDs quality of life measured by means of the QoL-AD, no significant between-group difference was found.

\section{Assessment of study quality according to the Drummond criteria}

Considering the methodological quality of the included studies, it can be acknowledged that the majority of 
articles gave a detailed description of the considered alternatives [see Additional file 2]. In most studies a comparison was made between a non-pharmacological intervention and CAU, whereas Davis et al. used a popular and widely available exercise program in order to reflect the actual practice in the respective community [33]. Two publications did not present detailed effectiveness results in the publication and the referenced articles could not be found [31,32].

\section{Perspective}

Three studies did not explicitly state the employed perspective [20, 30,31]. Considering the articles which stated their perspective, the most prevalent was the health and social care perspective, which is not taking into account informal care costs. Three studies reported both data from the health and social care perspective as well as from the societal perspective $[21,27,28]$. Nonetheless, the majority of identified studies also incorporated health outcomes of informal caregivers. Independent of the employed perspective some identified evaluations summed up the health and social care cost of both caregiver and PwD in order to reflect the effect on caregiver burden [20].

\section{Time horizon}

The time horizon of the economic analyses ranged from 3 up to 60 months. Considering the continuous progression of most types of dementia, data on long-term costeffectiveness would be important. Therefore, short-term analyses might not be able to capture the full consequences on health outcomes and resource utilization.

\section{Discussion}

This systematic review highlights recent evidence on health economic evaluations of non-pharmacological interventions for PwDs or PwMCI and their respective informal caregivers. To our knowledge this is the first systematic review focusing on psychosocial interventions directly delivered to patients or informal caregivers. Additionally, the methodological quality of the included health economic studies was evaluated according to the criteria by Drummond et al. [15].

In the light of the comparably low success rate in the development of AD drugs [39] and the limited number of agents in the current pipeline [40], the development of effective non-pharmacological interventions is of particular importance in the treatment and care of PwDs and the support of informal caregivers. This is also reflected in the increasing research conducted in this field [5] and the establishment of the pan-European research network INTERDEM aiming to promote collaboration and research on psychosocial interventions [6].
A previous systematic review detected a substantial lack of economic evidence on non-pharmacological interventions for PwDs [13]. In particular no economic evidence on physical exercise interventions could be identified by Knapp et al. [13].

In the meantime the evidence base has been slightly growing. The three identified studies concerned with exercise interventions showed that the programs were found to be potentially cost-effective for specific outcomes. An individually tailored home-based exercise program significantly delayed the deterioration of physical functioning of $\mathrm{AD}$ patients [20]. Further, an instructed walking program for PwDs and their caregivers is potentially cost-effective compared to CAU, when focusing on the reduction of BPSD as the outcome of interest [28]. However, the underlying effectiveness evaluation based on a larger sample did not prove the effectiveness with regard to this outcome [35]. Therefore, further evaluations are needed.

A structured occupational therapy intervention was found to be cost-effective from the caregiver's perspective, as it led to a reduction in caregiving time [23]. A previous CEA on occupational therapy also demonstrated costeffectiveness from the societal perspective [41].

Considering interventions to support and enhance cognition of PwDs, the evidence identified within this review is inconsistent. Joint reminiscence groups for PwDs and caregivers are found unlikely to be cost-effective compared to CAU [26]. The same findings were made for a carer-led CST intervention [27]. In contrast, a previous study conducted by Knapp et al. [42] found CST to be potentially cost-effective in comparison to CAU, considering its effects on cognition and quality of life. Likewise our systematic review found evidence that a long-term maintenance CST is potentially cost-effective [21].

With regard to psychological and behavioral treatments, self-management group rehabilitation for PwDs and their spouses [24] as well as cognitive-behavioral therapy for PwD-caregiver dyads [25], demonstrated effectiveness and cost-neutrality. In contrast, an intensive, multicomponent, semi-tailored psychosocial intervention program with counselling, education and support program was not found to be cost effective from the societal perspective [22].

Another previous systematic review by Jones et al. [14] focused on economic evaluations assessing interventions targeted at informal caregivers of community-dwelling PwDs. The authors indicated a lack of evidence and the need to gather carer data alongside patient data. In the framework of this previous review, a social support intervention comprising a facilitated access to a befriender volunteer was unlikely to be cost-effective [43]. Moreover, a home or telephone-based problem-solving therapy improved coping skills for a subgroup of 
caregivers without a significant difference in caregiver expenditures [44]. The evidence with regard to interventions directed at informal caregivers identified within this systematic review was also mixed. A manual-based individual coping program for informal caregivers was found to be cost-effective in the short and long term $[18,19]$. Further, an educational and support intervention appears to be effective and cost neutral [30]. In contrast, a family meetings intervention is not considered to be cost-effective compared to CAU [29].

\section{Methodological challenges of economic evaluations of dementia populations}

Regarding the methods of economic evaluations, the researchers of the identified trials most frequently applied CEA. As cost effectiveness measures, for instance, the incremental cost per one-point difference in NPI score [28] or per QoL-AD score $[18,21]$ were used. However, no cost-effectiveness thresholds exist for outcomes such as the change in the NPI or the MMSE score [45].

Moreover, the assessment of HRQoL in PwDs may be prone to recall bias and missing values are likely to occur within a cognitively impaired population [46]. Regarding proxy measures, a Dutch study conducted by Arons et al. indicates that patient-by-proxy HRQoL values must be interpreted cautiously, as caregivers are found to reflect parts of their own HRQoL onto the care recipient [47].

The CUAs that were included in this systematic review derived QALY of the PwD from dementia-specific instruments such as the DEMQOL and DEMQOL Proxy. Besides, generic proxy-rated caregiver EQ-5D values were employed to assess PwD's HRQoL. Concerning the EQ-5D, a study of Aguirre and colleagues [48] suggests that the EQ-5D may not capture all relevant aspects associated with dementia and PwD's experiences. However, Aguirre et al. [48] showed that compared to the dementia-specific measures DEMQOL and the Qol-AD, the EQ-5D demonstrated adequate psychometric properties and good reliability within this population. In addition, Orgeta et al. [49] indicated that the EQ-5D enables PwDs in mild and moderate stages to rate their own HRQoL. However, the study found significant difference between caregiver-rated and self-rated HRQoL. The ratings were influenced by the type of the caregiving relationship. Children of PwDs rated the HRQoL lower in comparison to spousal caregivers. Therefore, the authors recommend to use both self and proxy ratings in economic evaluations of interventions for PwDs [49].

In contrast, Algar and colleagues [50] propose observational measures for the effectiveness assessment of non-pharmacological interventions for PwDs. Especially, in more severe levels of dementia observational methods may provide additional valuable data and might be an alternative way to capture PwD's experiences [50]. The use of observational tools in health economic analyses might therefore be a valuable field of research.

There are as well challenges arising with regard to the identification and valuation of costs. A systematic review on cost-of-illness studies showed that the main cost drivers in the dementia care context are informal care costs [51]. Moreover, Wimo et al. [52] point to the complex interaction between those who may benefit from the consequences of the interventions and those who finance the care. The societal perspective includes all relevant cost irrespective where they occur and where they are financed. Therefore, Wimo and colleagues [52] recommend that taking on a societal perspective is often preferable.

Another systematic review by Krol et al. [53] assessed the effect of the inclusion and exclusion of informal care cost and effects on cost-effectiveness outcomes. The authors concluded that the inclusion of informal care can have a considerable impact on cost-effectiveness outcomes [53]. Moreover, Shearer et al. [54] recommend that the methods employed to measure and value informal care should be explicitly stated. In addition, sensitivity analyses should be employed to show the variability of the results using alternative methods [54].

\section{Limitations}

Even though a comprehensive literature search based on broad search terms was conducted, some papers meeting the search criteria might not have been identified. Moreover, this systematic review may be subject to a language bias, as solely publications in English and German were included.

A narrative synthesis was conducted due to the heterogeneity of the identified studies with regard to the employed health outcomes, perspectives of the analyses and time horizons. Furthermore, the study populations under examination comprised different types and severity levels of dementia. Moreover, the generalizability of the findings is limited since the identified studies were conducted in North American and European countries, with different underlying health and social care systems.

\section{Implications for future research}

There is evidence on the effectiveness of multicomponent psychosocial interventions on the maintenance of caregivers' psychological health [55] and delayed institutionalization of PwDs [12]. Furthermore, a recent study by Straubmeier et al. [56] shows a highly promising effect of a structured multicomponent intervention targeted at PwMCI and persons with mild to moderate dementia on their cognitive abilities and activities of daily living [56]. Future research should therefore examine the cost-effectiveness of multicomponent interventions and consider subgroups of $\mathrm{PwDs}$ at different 
disease stages. Moreover, the homogeneity of the respective caregivers should be taken into account.

Selected non-pharmacological interventions are recommended by most guidelines. However, there is still a lack of information on their dissemination as well as on facilitators and barriers of the translation of evidence on non-pharmacological interventions into practice.

\section{Conclusions}

This review provides evidence on economic aspects of non-pharmacological interventions in the therapeutic field of dementia. Health economic evaluations suggest that exercise programs, occupational therapy as well as cognitive and psychological interventions directly delivered to PwDs demonstrate cost-effectiveness compared to CAU with regard to specific outcomes. No economic evaluations on sensory interventions and creative interventions such as art, music or dance therapy could be identified.

\section{Additional files}

Additional file 1: Search strategies and search results. (PDF $106 \mathrm{~kb}$ ) Additional file 2: Quality assessment of included studies according to the 10-item Drummond check-list. (PDF 199 kb)

\section{Abbreviations}

AChEl: Acetylcholinesterase inhibitor; AD: Alzheimer's disease; ADASCog: Alzheimer's Disease Assessment Scale-Cognition Subscale; ADCSADL: Alzheimer's Disease Cooperative Study-Activities of Daily Living Inventory; BPSD: Behavioral and psychological symptom of dementia; CAU: Care as usual; CBT: Cognitive-behavioral therapy; CEA: Cost-effectiveness analysis; CEAC: Costeffectiveness acceptability curve; Cl: Confidence Interval; CSDD: Cornell Scale for Depression in Dementia; CSRI: Client Service Receipt Inventory; CST: Cognitive stimulation therapy; CUA: Cost-utility analysis; DSM-IV: Diagnostic and Statistical Manual of Mental Disorders, 4th Edition; EQ-5D: EuroQoL 5-dimensions; FIM: Functional Independence Measure; FINALEX: Finnish Alzheimer disease exercise trial; GHQ: General Health Questionnaire; HADS: Hospital Anxiety and Depression Scale; HADS-T: Hospital Anxiety and Depression Scale (total score); HRQoL: Health-Related Quality of Life; IADL: Instrumental Activities of Daily Living; ICD-10: International statistical classification of diseases and related health problems, 10th revision; ICER: Incremental cost-effectiveness ratio; iCST: Individual cognitive stimulation therapy; ICUR: Incremental cost-utility ratio; MCST: Maintenance cognitive stimulation therapy; MINI: Mini International Neuropsychiatric Interview; MMSE: Mini-Mental Status Examination; NHS: National Health Service; NPI: Neuropsychiatric Inventory; OOP: Out-of-pocket; PwD: Person with dementia; PwMCI: Person with mild cognitive impairment; QALY: Qualityadjusted life-years; QCPR: Quality of Caregiver - Patient Relationship; QoL: Quality of Life; QoL-AD: Quality of Life in Alzheimer's Disease scale; RAID: Rating Anxiety in Dementia scale; RAND 36: RAND 36-Item Health Survey; RCT: Randomized controlled trial; RUD: Resource Utilization in Dementia; SCQ: Sense of Competence Questionnaire; SF-12: Short Form questionnaire-12 items; SPPB: Short Physical Performance Battery; ZBI: Zarit Caregiver Burden Interview

\section{Funding}

This research work was funded by the Karl and Veronica CarstensFoundation as part of the National Graduate College 'Optimisation strategies in Dementia - OptiDem' (Lead: Prof. Dr. Elmar Gräßel). The Karl and Veronica Carstens-Foundation had no role in study design, data collection and analysis, decision to publish, or preparation of the manuscript.

\section{Availability of data and materials}

All data generated or analyzed during this study are included in this published article [and its supplementary information files].

\section{Disclosure}

This work was performed in partial fulfillment of the requirements for obtaining the degree 'Dr. rer. biol. hum.' for author FN at the Medical Faculty of the Friedrich-Alexander-University Erlangen-Nürnberg (FAU).

\section{Authors' contributions}

FN made substantial contributions to the concept and design of the review and conducted all literature searches. FN performed the acquisition of data as first reviewer during the PRISMA process, analyzed and interpreted the data, and wrote the manuscript. JB was the second reviewer in the PRISMA process, made substantial contributions to the analysis and interpretation of the included studies and was involved in revising the manuscript critically for important intellectual content. PKR made substantial contributions to the concept and design of the review and was involved in revising the manuscript critically for important intellectual content. Moreover, PKR was the third reviewer in the study selection process. All authors commented on and approved the final manuscript.

\section{Ethics approval and consent to participate}

Not applicable.

Consent for publication

Not applicable.

Competing interests

The authors declare that they have no competing interests.

\section{Publisher's Note}

Springer Nature remains neutral with regard to jurisdictional claims in published maps and institutional affiliations.

Received: 18 July 2017 Accepted: 16 February 2018

Published online: 09 March 2018

References

1. Prince M, Wimo A, Guerchet M, Ali G-C, Wu Y-T, Prina M. World Alzheimer Report 2015: The global impact of dementia. An analysis of prevalence, incidence, cost and trends. London: Alzheimer's Disease International; 2015.

2. Versijpt J. Effectiveness and cost-effectiveness of the pharmacological treatment of Alzheimer's disease and vascular dementia. J Alzheimers Dis. 2014:42(Suppl 3):S19-25.

3. Ghezzi L, Scarpini E, Galimberti D. Disease-modifying drugs in Alzheimer's disease. Drug Design, Development and Therapy. 2013;7:1471-9.

4. Livingston G, Sommerlad A, Orgeta V, Costafreda SG, Huntley J, Ames D, Ballard C, Banerjee S, Burns A, Cohen-Mansfield J, et al. Dementia prevention, intervention, and care. Lancet (London, England). 2017; 390(10113):2673-734.

5. Oyebode JR, Parveen S. Psychosocial interventions for people with dementia: an overview and commentary on recent developments. Dementia. 2016:1471301216656096.

6. Moniz-Cook E, Vernooij-Dassen M, Woods B, Orrell M. Psychosocial interventions in dementia care research: the INTERDEM manifesto. Aging Ment Health. 2011;15(3):283-90

7. Farina N, Rusted J, Tabet N. The effect of exercise interventions on cognitive outcome in Alzheimer's disease: a systematic review. Int Psychogeriatr. 2014; 26(1):9-18.

8. Groot C, Hooghiemstra AM, Raijmakers PG, van Berckel BN, Scheltens P, Scherder EJ, van der Flier WM, Ossenkoppele R. The effect of physical activity on cognitive function in patients with dementia: a meta-analysis of randomized control trials. Ageing Res Rev. 2016;25:13-23.

9. Forbes D, Forbes SC, Blake CM, Thiessen EJ, Forbes S. Exercise programs for people with dementia. The Cochrane database of systematic reviews. 2015; 4:Cd006489.

10. Orgeta V, Qazi A, Spector AE, Orrell M. Psychological treatments for depression and anxiety in dementia and mild cognitive impairment. The Cochrane database of systematic reviews. 2014;1:Cd009125. 
11. Enache D, Winblad B, Aarsland D. Depression in dementia: epidemiology, mechanisms, and treatment. Current opinion in psychiatry. 2011;24(6):461-72.

12. Olazaran J, Reisberg B, Clare L, Cruz I, Pena-Casanova J, Del Ser T, Woods B, Beck C, Auer S, Lai C, et al. Nonpharmacological therapies in Alzheimer's disease: a systematic review of efficacy. Dement Geriatr Cogn Disord. 2010; 30(2):161-78.

13. Knapp M, lemmi V, Romeo R. Dementia care costs and outcomes: a systematic review. Int J Geriatr Psychiatry. 2013;28(6):551-61.

14. Jones C, Edwards RT, Hounsome B. A systematic review of the costeffectiveness of interventions for supporting informal caregivers of people with dementia residing in the community. Int Psychogeriatr. 2012;24(1):6-18.

15. Drummond MF, Sculpher MJ, Torrance GW, O'Brien BJ, Stoddart GL. Methods for the Economic Evaluation of Health Care Programmes. Third Edition. London: Oxford University Press; 2005

16. Zweifel P, Breyer F, Kifmann M. Health Economics. 2nd ed. Berlin and Heidelberg: Springer-Verlag; 2009.

17. Centre for Reviews and Dissemination: Systematic Reviews. CRD's guidance for undertaking reviews in health care, University of York; 2009.

18. Livingston G, Barber J, Rapaport P, Knapp M, Griffin M, King D, Romeo R Livingston D, Mummery C, Walker Z, et al. Long-term clinical and costeffectiveness of psychological intervention for family carers of people with dementia: a single-blind, randomised, controlled trial. The Lancet Psychiatry. 2014;1(7):539-48.

19. Livingston G, Barber J, Rapaport P, Knapp M, Griffin M, Romeo R, King D, Livingston D, Lewis-Holmes E, Mummery C, et al. START (STrAtegies for RelaTives) study: a pragmatic randomised controlled trial to determine the clinical effectiveness and cost-effectiveness of a manual-based coping strategy programme in promoting the mental health of carers of people with dementia. Health technology assessment (Winchester, England). 2014; 18(61):1-242.

20. Pitkälä KH, Poysti MM, Laakkonen ML, Tilvis RS, Savikko N, Kautiainen $H_{\text {, }}$ Strandberg TE. Effects of the Finnish Alzheimer disease exercise trial (FINALEX): a randomized controlled trial. JAMA Intern Med. 2013;173(10): 894-901.

21. D'Amico F, Rehill A, Knapp M, Aguirre E, Donovan H, Hoare Z, Hoe J, Russell IS, A. Streater A. Whitaker C et al: Maintenance cognitive stimulation therapy: an economic evaluation within a randomized controlled trial. J Am Med Dir Assoc 2015, 16(1):63-70.

22. Sogaard R, Sorensen J, Waldorff FB, Eckermann A, Buss DV, Phung KT, Waldemar G. Early psychosocial intervention in Alzheimer's disease: cost utility evaluation alongside the Danish Alzheimer's intervention study (DAISY). BMJ Open. 2014:4(1):e004105.

23. Gitlin LN, Hodgson N, Jutkowitz E, Pizzi L. The cost-effectiveness of a nonpharmacologic intervention for individuals with dementia and family caregivers: the tailored activity program. Am J Geriatr Psychiatr. 2010;18(6):510-9.

24. Laakkonen ML, Kautiainen H, Holtta E, Savikko N, Tilvis RS, Strandberg TE, Pitkälä KH. Effects of self-management groups for people with dementia and their spouses-randomized controlled trial. J Am Geriatr Soc. 2016;64(4):752-60.

25. Spector A, Charlesworth G, King M, Lattimer M, Sadek S, Marston L, Rehill A Hoe J, Qazi A, Knapp M, et al. Cognitive-behavioural therapy for anxiety in dementia: pilot randomised controlled trial. Br J Psychiatry. 2015;206(6):509-16.

26. Woods RT, Bruce E, Edwards RT, Elvish R, Hoare Z, Hounsome B, Keady J, Moniz-Cook ED, Orgeta V, Orrell M, et al. REMCARE: Reminiscence groups for people with dementia and their family caregivers - Effectiveness and costeffectiveness pragmatic multicentre randomised trial. Health Technology Assessment. 2012;16(48):V-vii.

27. Orgeta V, Leung P, Yates L, Kang S, Hoare Z, Henderson C, Whitaker C, Burns A, Knapp M, Leroi I, et al. Individual cognitive stimulation therapy for dementia: a clinical effectiveness and cost-effectiveness pragmatic, multicentre, randomised controlled trial. Health Technol Assess. 2015;19(64):7-73.

28. D'Amico F, Rehill A, Knapp M, Lowery D, Cerga-Pashoja A, Griffin M, lliffe S, Warner J. Cost-effectiveness of exercise as a therapy for behavioural and psychological symptoms of dementia within the EVIDEM-E randomised controlled trial. International Journal of Geriatric Psychiatry. 2016:31(6):656-65.

29. Joling KJ, Bosmans JE, van Marwijk HWJ, van der Horst HE, Scheltens P, Vroomen JLM, van Hout HPJ. The cost-effectiveness of a family meetings intervention to prevent depression and anxiety in family caregivers of patients with dementia: A randomized trial. Trials. 2013;14(1) no pagination

30. Dahlrup B, Nordell E, Steen Carlsson K, Elmstahl S. Health economic analysis on a psychosocial intervention for family caregivers of persons with dementia. Dement Geriatr Cogn Disord. 2013;37(3-4):181-95.
31. Wray LO, Shulan MD, Toseland RW, Freeman KE, Vasquez BE, Gao J. The effect of telephone support groups on costs of care for veterans with dementia. The Gerontologist. 2010;50(5):623-31.

32. Van Houtven $\mathrm{CH}$, Thorpe JM, Chestnutt D, Molloy M, Boling JC, Davis LL. Do nurse-led skill training interventions affect informal caregivers' out-of-pocket expenditures? The Gerontologist. 2013;53(1):60-70.

33. Davis JC, Bryan SM, Marra CA, Sharma D, Chan A, Beattie BL, Graf P, LiuAmbrose T. An Economic Evaluation of Resistance Training and Aerobic Training versus Balance and Toning Exercises in Older Adults with Mild Cognitive Impairment. PLoS ONE. 2013;8(5) no pagination

34. Prince M, Bryce R, Ferri C. World Alzheimer Report 2011: The benefits of early diagnosis and intervention. London: Alzheimer's Disease International; 2011.

35. Lowery D, Cerga-Pashoja A, lliffe S, Thuné-Boyle I, Griffin M, Lee J, Bailey A, Bhattacharya R, Warner J. The effect of exercise on behavioural and psychological symptoms of dementia: the EVIDEM-E randomised controlled clinical trial. International journal of geriatric psychiatry. 2014;29:819-27.

36. Orrell M, Aguirre E, Spector A, Hoare Z, Woods RT, Streater A, Donovan H, Hoe J, Knapp M, Whitaker C, et al. Maintenance cognitive stimulation therapy for dementia: single-blind, multicentre, pragmatic randomised controlled trial. Br J Psychiatr. 2014;204(6):454-61.

37. Waldemar G, Waldorff FB, Buss DV, Eckermann A, Keiding N, Rishøj S, Siersma V, Sørensen J, Sørensen LV, Vogel A. The Danish Alzheimer intervention study: rationale, study design and baseline characteristics of the cohort. Neuroepidemiology. 2011;36(1):52-61.

38. Knapp M, King D, Romeo R, Schehl B, Barber J, Griffin M, Rapaport P, Livingston D, Mummery C, Walker Z, et al. Cost effectiveness of a manual based coping strategy programme in promoting the mental health of family carers of people with dementia (the START (STrAtegies for RelaTives) study): a pragmatic randomised controlled trial. BMJ (Clinical research ed). 2013;347:f6342.

39. Cummings JL, Morstorf T, Zhong K. Alzheimer's disease drug-development pipeline: few candidates, frequent failures. Alzheimers Res Ther. 2014;6(4):37.

40. Cummings J, Morstorf T, Lee G. Alzheimer's drug-development pipeline: 2016. Alzheimer's \& Dementia: Translational Research \& Clinical Interventions. 2016;2(4):222-32.

41. Graff MJ, Adang EM, Vernooij-Dassen MJ, Dekker J, Jonsson L, Thijssen M, Hoefnagels WH, Rikkert MG. Community occupational therapy for older patients with dementia and their care givers: cost effectiveness study. BM (Clin Res ed). 2008;336(7636):134-8.

42. Knapp M, Thorgrimsen L, Patel A, Spector A, Hallam A, Woods B, Orrell M. Cognitive stimulation therapy for people with dementia: cost-effectiveness analysis. The British journal of psychiatry : the journal of mental science. 2006;188:574-80.

43. Charlesworth G, Shepstone L, Wilson E, Thalanany M, Mugford M, Poland F: Does befriending by trained lay workers improve psychological well-being and quality of life for carers of people with dementia, and at what cost? A randomised controlled trial. Health technology assessment (Winchester, England) 2008, 12(4):iii, v-ix, 1-78.

44. Roberts J, Browne G, Milne C, Spooner L, Gafni A, Drummond-Young M, LeGris J, Watt S, LeClair K, Beaumont L, et al. Problem-solving counseling for caregivers of the cognitively impaired: effective for whom? Nurs Res. 1999; 48(3):162-72.

45. Livingston G, Kelly L, Lewis-Holmes E, Baio G, Morris S, Patel N, Omar RZ, Katona C, Cooper C. A systematic review of the clinical effectiveness and cost-effectiveness of sensory, psychological and behavioural interventions for managing agitation in older adults with dementia. Health technology assessment (Winchester, England). 2014;18(39):1-226. v-vi

46. Davis JC, Bryan S, Marra CA, Hsiung G-YR, Liu-Ambrose T. Challenges with cost-utility analyses of behavioral interventions among older adults at risk for dementia. Br J Sports Med. 2015;49(20):1343-7.

47. Arons AM, Krabbe PF, Scholzel-Dorenbos CJ, van der Wilt GJ, Rikkert MG Quality of life in dementia: a study on proxy bias. BMC Med Res Methodol. 2013;13:110.

48. Aguirre E, Kang S, Hoare Z, Edwards RT, Orrell M. How does the EQ-5D perform when measuring quality of life in dementia against two other dementia-specific outcome measures? Quality of life research : an international journal of quality of life aspects of treatment, care and rehabilitation. 2016;25(1):45-9.

49. Orgeta V, Edwards RT, Hounsome B, Orrell M, Woods B. The use of the EQ$5 \mathrm{D}$ as a measure of health-related quality of life in people with dementia and their carers. Quality of life research : an international journal of quality of life aspects of treatment, care and rehabilitation. 2015;24(2):315-24. 
50. Algar K, Woods RT, Windle G. Measuring the quality of life and well-being of people with dementia: a review of observational measures. Dementia (London, England). 2016;15(4):832-57.

51. Schaller S, Mauskopf J, Kriza C, Wahlster P, Kolominsky-Rabas PL. The main cost drivers in dementia: a systematic review. International Journal of Geriatric Psychiatry. 2015;30(2):111-29.

52. Wimo A, Jönsson L, Winblad B: Health economic aspects of dementia. In: Dementia, Fifth Edition. Edited by David Ames, Alistair Burns, O'Brien JT, vol. 5. Boca Raton: CRC Press; 2017.

53. Krol M, Papenburg J, van Exel J. Does including informal care in economic evaluations matter? A systematic review of inclusion and impact of informal care in cost-effectiveness studies. PharmacoEconomics. 2015;33(2):123-35.

54. Shearer J, McCrone P, Romeo R. Economic evaluation of mental health interventions: a guide to costing approaches. PharmacoEconomics. 2016; 34(7):651-64.

55. Dickinson C, Dow J, Gibson G, Hayes L, Robalino S, Robinson L. Psychosocial intervention for carers of people with dementia: what components are most effective and when? A systematic review of systematic reviews. Int Psychogeriatr. 2016;29(1):31-43.

56. Straubmeier M, Behrndt EM, Seidl H, Özbe D, Luttenberger K, Graessel E. Non-pharmacological treatment in people with cognitive impairment. Deutsches Arzteblatt international. 2017:114(48):815-21.

\section{Submit your next manuscript to BioMed Central} and we will help you at every step:

- We accept pre-submission inquiries

- Our selector tool helps you to find the most relevant journal

- We provide round the clock customer support

- Convenient online submission

- Thorough peer review

- Inclusion in PubMed and all major indexing services

- Maximum visibility for your research

Submit your manuscript at www.biomedcentral.com/submit 